\title{
Computational Fluid Dynamics in Unruptured Intracranial Aneurysms
}

\author{
Maruf Matmusaev ${ }^{1}$, Yasuhiro Yamada ${ }^{2}$, Tsukasa Kawase ${ }^{2}$, Riki \\ Tanaka $^{2}$, Miyatani Kyosuke ${ }^{2}$, Yoko Kato ${ }^{2}$, Ahmed Ansari ${ }^{2}$ \\ ${ }^{1}$ Republican Specialized Scientific and Practical Medical Center of Neurosurgery, Tashkent, \\ UZBEKISTAN \\ ${ }^{2}$ Department of Neurosurgery, Banbuntane Hotokukai Hospital, Fujita Health University, \\ Nagoya, JAPAN
}

\begin{abstract}
Introduction and Objective: Intracranial aneurysm, also known as brain aneurysm, is a cerebrovascular disorder in which weakness in the wall of a cerebral artery causes a localized dilation or ballooning of the blood vessel. There is no objective way, device or tools, of predicting rupture of aneurysm so far. Computational fluid dynamics (CFDs) was proposed as a tool to identify the rupture risk. Purpose of study: To reveal the correlation of CFD findings with intraoperative microscopic findings and prove the relevance of CFDin the prediction of rupture risk and in the management of unruptured intracranial aneurysms. Subjects and Methods: A prospective cohort study was conducted inNeurosurgery department of Fujita Health University Banbuntane Hotokukai Hospital, Nagoya, Japanduring a 3-month period in 2018,from January to March, Ten patientswere diagnosed unruptured intracranial aneurysms (UIA). In diagnosis computed tomography (CT) angiogram, CFD and digital subtraction angiogram were included. Intraoperatively microscopic examination of the aneurysm wall was carried out and images recorded. The correlation between microscopic dome morphology and CFD information was performed. Results: Nine cases were found intraoperatively to have a higher risk of rupture based on the thinning of the wall. One cases had an atherosclerotic wall. All cases had low wall shear stress (WSS). In $90 \%$ of cases Low WSS was able to predict the potency rupture risk in the near future. Conclusions: This study of CFD and its correlation with intraoperativefindings of the aneurysm suggested that low WSS of the aneurysm wall is associated with thin wall aneurysm and hence increased risk of aneurysm rupture. Thus CFD can be used to predict the risk of rupture of unruptured aneurysm and for planning of its treatment.
\end{abstract} Key words: computational flow dynamics, intraoperative microscopic findings, unruptured intracranial aneurysms 


\section{Introduction}

Intracranial aneurysm, also known as brain aneurysm, is a cerebrovascular disorder in which weakness in the wall of a cerebral artery causes a localized dilation or ballooning of the blood vessel. Nowadays about $1 \%-5 \%$ of adult population around the globe suffer from an aneurysm and itcomplications [1,2], varying between $<1 \%$ in young adults to $4 \%$ in the elderly.[4] The fatal outcomefrom a ruptured aneurysm is quite high and in patients surviving it, the morbidity is high.[1,3] It is difficult to determine which aneurysm will rupture in the future.[6] The management of unruptured aneurysms did not prevented from complications. The morbidity and mortality can be as high as $10 \%$ and $2.5 \%$, respectively.[7]

Understanding aneurysm pathogenesis and the patient-specific risk factors associated with rupture would, therefore, be useful in clinical decision-making. Several approaches have been considered, including the identification of morphological, histological, and hemodynamic features associated with aneurysm growth and subsequent rupture, with an increasing trend toward the use of CFD in evaluating the role of hemodynamic shear forces on the arterial endothelium. [5,18,19,20,14].

These days, some researches regarding using CFD to predict the rupture chances of an unruptured aneurysm were conducted. CFD, a branch of fluid mechanics in mechanical engineering, has been in use in industry in aerodynamics, hydrodynamics of vehicles and a lot of other fields.[8,9,10].It uses numerical analysis and algorithms to analyze and solve problems that involve fluid flows. Itsuse in biomedical engineering has progressed only in the lastdecade. Many simulations have been used to study carotid andintra-cranial cerebrovascular diseases. We were interested inits utilization in cerebral aneurysms in specific.

CFD is a device used to study the hemodynamics of an aneurysm and predict the risk of rupture of an aneurysm. There are many opinions and controversies about the interpretation of CFD. While some advocate that lower wall shear stress (WSS) increases rupture risk others propose that higher WSS increase the rupture risk. Our researches based on comparison of the preoperative CFD images and the intraoperative microscopic images of the aneurysm and reveal whether there is correlation and any predictive value for CFD.

\section{Materials and Methods}

A prospective cohort study was conducted during a 3-month period in 2018, from January to March, in ten patients with unruptured intracranial aneurysms using computed tomography (CT) angiogram, CFD and digital subtraction angiogram. Patient consent was taken for the study. The cases selected for surgery were the ones which had increased rupture risk as per CFD findings. The CFD software used was Hemoscope 2 (Ziosoft corporation, Minato ward, Tokyo, Japan). CFD codes contain three main components (1) a preprocessor, (2) a solver, and (3) a postprocessor. 


\section{Preprocessor}

Preprocessing consists of inputting a fluid flow problem into a CFD program. Most of the time spent is devoted to this process.

\section{Solver}

Numerical solution techniques are available such as finite difference, finite element, finite volume, and spectral methods.

\section{Postprocessor}

CFD analysis requires knowledge of the volumetric region traversed by the fluid (i.e., aneurysm including connected surrounding vasculature) plus information about velocity and pressure of the fluid at the boundaries of the chosen region of interest (boundary conditions).

The object of this process is to visualize the computational results. The changes in blood flow profiles, pressure distribution, WSS, oscillating shear index, and shear rate can be visualized using color rendering techniques. The WSS and pressure at various places in the aneurysm sac can be calculated.

Focal higher pressure with the translucent thinner regions of the aneurysm wall suggest potentially elevated wall tension, which is approximately inversely proportional to wall thickness and proportional to transmural pressure.

On CFD Areas with thin wall - low WSS, high pressure, parallel vectors, spiral flow signifies a growing aneurysm with a possible rupture.
WSS is the frictional force applied by flowing blood tangentially on the vessel wall. Time-Averaged Wall Shear Stress (TAWSS) taken for one cardiac cycle as WSS can change with cardiac systole and diastole. The WSS mentioned below is the TAWSS.

We use generic inflow and outflow boundary conditions because patient-specific flow waveforms are generally unavailable. Model geometries are produced by using available imaging data and hence are limited by the resolution of the medical scanners.

We reveal that in five out of ten cases were internal carotid artery, four cases were middle cerebral artery aneurysm and another one anterior communicating artery (Acom) aneurysm [Table 1].

The patients were operated, and the aneurysm wall was observed with a microscope. Intraoperatively decreased vessel wall thinness was determined by the transparency of the vessel wall and the presence of a bleb on the aneurysm wall using microscope or ICG-VA visualization of brain vessels anterior circulation. Yellowish color of the vessel wall indicates atherosclerosis. Ability to see blood flow in the aneurysm or thinning of the vessel wall, i.e., translucency, and bleb on aneurysm wall were predictors for future rupture. Intraoperatively, indocyanine video angiography (ICG-VA) was also used. The areas of thinning had higher fluorescence and flow of blood could also be noted with ICG-VA. 
Table 1 - Aneurysm data

\begin{tabular}{|c|c|c|c|c|c|c|}
\hline Sex & Age & $\begin{array}{c}\text { Type of } \\
\text { aneurysm }\end{array}$ & $\begin{array}{c}\text { pressure } \\
(\mathrm{mmHg})\end{array}$ & WSS & Vessel wall character intraoperatively & Bleb-present/absent \\
\hline Female & 66 & MCA & 99.78 & Low & Thinning of the aneurysmwall & Bleb on dome \\
\hline Male & 54 & IC-PC & 99.57 & Low & Thick wall of the aneurysm & - \\
\hline Female & 62 & IC-PC & 99.64 & Low & $\begin{array}{c}\text { Thin wall of the dome and thick wall } \\
\text { of the neck }\end{array}$ & - \\
\hline Female & 75 & MCA & 99.93 & Low & Thin wall of the aneurysm & - \\
\hline Female & 48 & ICA & 100.01 & Low & Thin wall of the aneurysm & - \\
\hline Female & 71 & MCA & 100.15 & Low & Thin wall of the aneurysm & - \\
\hline Female & 87 & IC-PC & 99.6 & Low & $\begin{array}{c}\text { Thin of the wall dome and thick wall } \\
\text { of the neck }\end{array}$ & - \\
\hline Female & 77 & Acom & 99.8 & Low & Thin and thick wall of the aneurysm & - \\
\hline Female & 67 & ICA & 100.1 & Low & Thin wall of the aneurysm & - \\
\hline Male & 67 & MCA & 99.7 & Low & Thin wall of the aneurysm & - \\
\hline
\end{tabular}

WSS - Wall shear stress; Acom - Anterior communicating artery; IC-PC - Internal carotid-posterior communicating artery; MCA - Middle cerebral artery; ICA - Internal carotid artery

\section{Results}

CFD studies was done in all the 10 cases which revealed low WSS in all the cases suggesting thin walled aneurysm. However intraoperativly only 9 cases were thin walled.
Thus CFD predicted correctly $90 \%$ of the cases. Intraoperativly one case had an atherosclerotic plaque involving the dome of the aneurysm.

In all cases, the pressure in the aneurysm was increased whereas the WSS was low. 


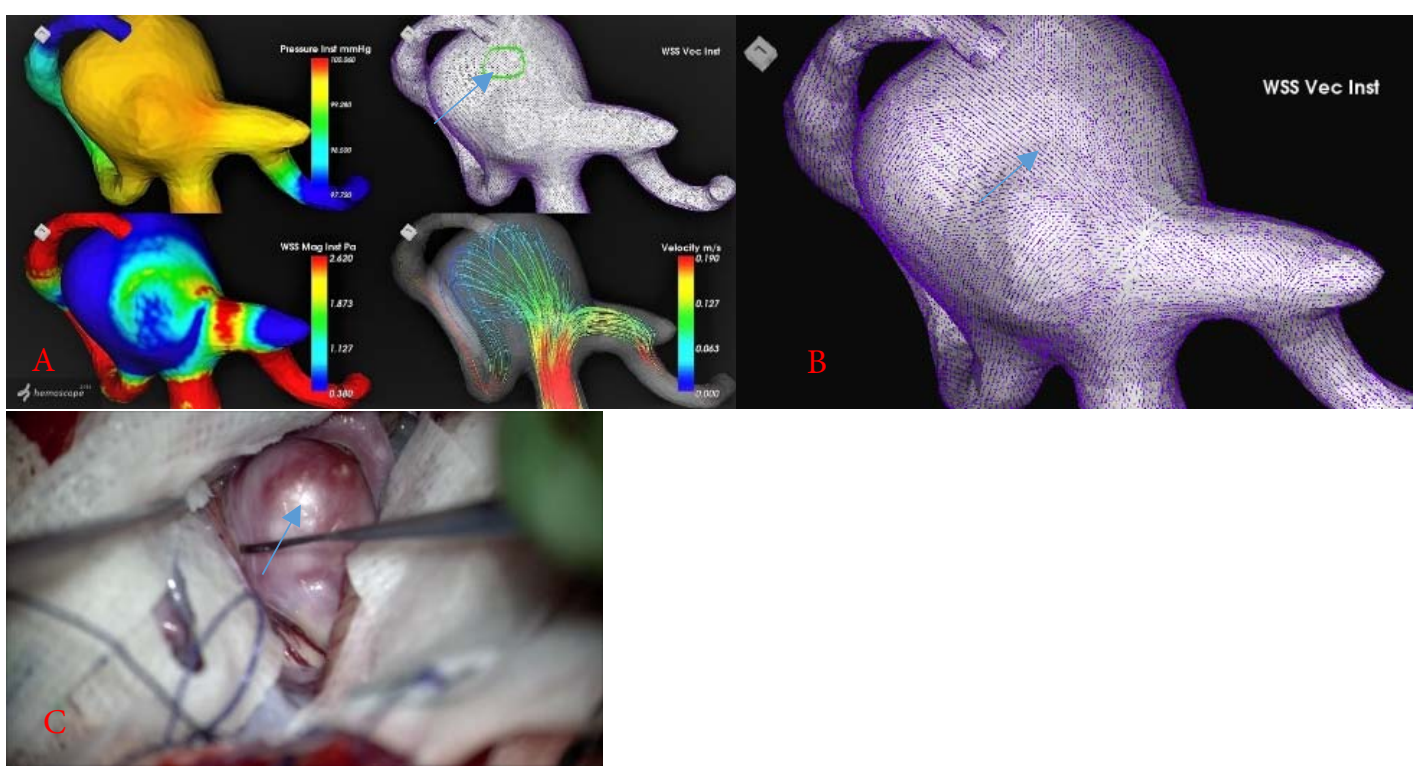

Figure 1-A,B- Computational fluid dynamics image of a middle cerebral artery aneurysm with high pressure in aneurysm dome and low wall shear stress, C- Intraoperative image of the same aneurysm with thinned wall.Arrow:there are parallel vectors which indicate area with thin walled aneurysm.

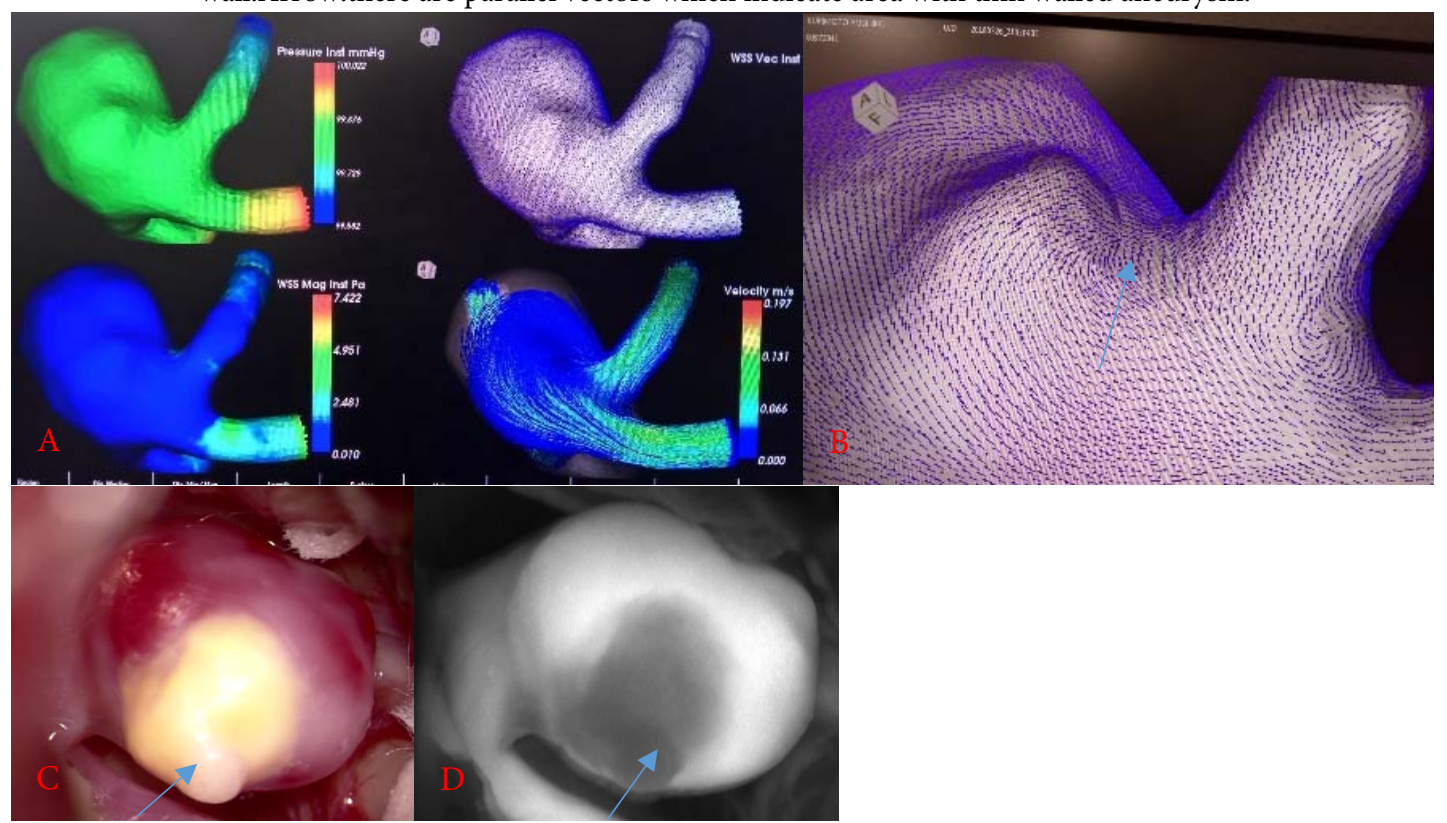

Figure 2 - A,B-Computational fluid dynamics image of a Acom aneurysm with middle pressure in aneurysm dome and low wall shear stress. C, D- Intraoperative image of the same aneurysm having thin wall with an atherosclerotic bleb. Filling of ICG is not seen well in the region of thick atherosclerotic wall. Arrow: Divergent vectors indicatethick walled aneurysm. 


\section{Discussion}

Improvement in the imaging and screening modalities has contributed to the increase in detection of unruptured aneurysms. In some countries, the incidence of intracranial aneurysms is more than the average mentioned in the studies. There is a need to quantify objectively the risk of rupture in the cohort of people with incidentally found aneurysms.

CFD which was earlier a purely engineering tool has comeinto vogue in biomedical engineering with its usefulness inknowing the flow dynamics in an aneurysm noninvasively.CFD helps in calculating the velocity of blood flowing in theaneurysm sac, the pressure in the aneurysm sac and the WSS.

There are many laboratory studies of CFD but they have alimitation that the wall in their studies is rigid and therebychange the outcome of studies. In vivo studies have been few.

However, there were no studies which could directly see if the results shown in CFD analysis could be correlated intraoperatively. Kadasi L.M et al observed the thinning of the aneurysmal wall in seven cases out of nine which is a predictor for future rupture.[11] Increased pressure in the aneurysm sac in all cases shows that hemodynamic factors have been responsible for the initiation and growth of the aneurysm.[12-15]

There is still no consensus regarding whether low WSS or high WSS contributes to aneurysm formation. Meng et al. proposed that high WSS correlates with Type laneurysm formation, i.e., small and transparent aneurysms, whereas low WSS contributed to Type 2 aneurysm formation, i.e., thick walled atherosclerotic type.[12] Cebral et al. reported that ruptured aneurysms have higher WSS compared with unruptured aneurysms.[13] Xiang et al. have proposed in their study that lower WSS contributes to rupture of aneurysm.[14]

Yoichi et al. in their study proposed that lower shear stress is associated with increased risk of rupture.[15] This controversy has mainly been due the low sample sizes in the studies and a small number of studies so far.

In our study, 9 out of 10 cases with low WSS had thinning of the vessel wall. One aneurysm had a bleb. Cebral JR et al have shown that thinning of vessel wall identified bythe transparency of vessel wall or bleb formation increases the risk of rupture.[16]

Results shown in CFD analysis highly correlated with intraoperative findings. In $90 \%$ of cases, we revealed thinning of the aneurysmal wall during operation which is a predictor for future rupture. Hence, using the CFD adjunct the microsurgery assist on choosing adequate microsurgical tactic taking into account predicted risk of aneurysm rupture.

This is in contrast to what Meng et al. have proposed. They proposed that higher WSS leads to thin wall and lower WSS leads to thick wall aneurysms.[12] So this shows that the mechanisms proposed so far in aneurysm initiation, growth, and rupture are far from accurate. However in all the cases, it has shown that the pressure in the aneurysm sac is high, which denotes increased risk. 
There has been no controversy as far the pressure parameter in CFD is considered. The WSS component is controversial with regard to the interpretation.

Laith Met al suggest a possible unifying mechanism for aneurysm progression to rupture, whereby low WSS is associated with thinning of the wall leading to a theoretical increased wall tension in these thin regions of the aneurysm wall.[17]

We accept the limitations of our study due to the small number of patients. While the controversies regarding WSS are not resolved, CFD does hold promise as an innovative tool in the management of aneurysm patients.

Until recently, the lack of reliable imaging techniques to quantify intraaneurysm blood flow in the human body, and the difficulty in measuring the hemodynamic parameters in vivo, have prevented researchers from investigating aneurysmal hemodynamics and its relation to growth and rupture mechanism in depth. Recent development in computational fluid dynamics (CFD), however, has given researchers a powerful tool in the investigation of intracranial aneurysms.

There are many advantages when considering CFD. CFD provides a means of simulating blood flow, in cerebral vasculature noninvasively. It provides detailed visual and comprehensive information colorfully. It provides the ability to know the pressure, WSS, streamlines and vector at various points in the aneurysm and helps us to know the character of blood flow in the aneurysm. Besides Low WSS correlate with the thickness of aneurism walls and assist in defining microsurgical management strategy

However, it has some disadvantages. There is difficulty in interpreting the results, software is available only at a few centers, insufficient resolution when using low resolution magnetic resonance imaging or $\mathrm{CT}$, numerical errors can occur during the test leading to incorrect conclusions and wrong management. Cooperation between the surgeon and the biomedical engineer is also difficult at times.

\section{Conclusions}

This study of CFD and it is correlation with intraoperative findings of the aneurysm suggested that low WSS of the aneurysm wall is associated with thin wall aneurysm and hence increased risk of aneurysm rupture. Thus CFD can be used to predict the risk of rupture of unruptured aneurysm and for planning of its treatment.

\section{References}

1. Brisman JL, Song JK, Newell DW. Cerebral aneurysms. N Engl J Med 2006;355:928-39

2. Vlak MH, Algra A, Brandenburg R, et al. Prevalence of unruptured intracranial aneurysms, with emphasis on sex, age, comorbidity, country, and time period: a systematic review and meta-analysis. Lancet Neurol 2011;10:626-36

3. Nieuwkamp DJ, Setz LE, Algra A, et al. Changes in case fatality of aneurysmal subarachnoid haemorrhage over time, according to age, sex, and region: a metaanalysis. Lancet Neurol 2009;8:635- 42

4. Komotar RJ, Mocco J, Solomon RA. Guidelines for the surgicaltreatment of unruptured intracranial aneurysms: The first annual J. Lawrence pool memorial research symposium - Controversies in the management of cerebral aneurysms. Neurosurgery 2008;62:183-93. 
5. Baharoglu MI, Schirmer CM, Hoit DA, Gao BL, Malek AM: Aneurysm inflow-angle as a discriminant for rupture in sidewall cerebral aneurysms: morphometric and computational fluid dynamic analysis. Stroke 41:1423-1430, 2010

6. Unruptured intracranial aneurysms - Risk of rupture and risks of surgical intervention. International Study of Unruptured Intracranial Aneurysms Investigators. N Engl J Med 1998;339:1725-33.

7. King JT Jr., Berlin JA, Flamm ES. Morbidity and mortality from elective surgery for asymptomatic, unruptured, intracranial aneurysms: A meta-analysis. J Neurosurg 1994;81:837-42.

8. Tu J, Yeoh GH, Liu C. Introduction. In: Yeoh GH, editor. Computational Fluid Dynamics: A Practical Approach. 2nd ed. Oxford: Elesevier; 2013. p. 1-29.

9. Anderson JD, Wendt J. Computational Fluid Dynamics. Heidelberg: Springer; 1995

10. Frank M. White., Fluid Mechanics, Potential Flow and Computational Fluid Dynamics. University of Rhode Island, p 590; 2011

11. Kadasi LM, Dent WC, Malek AM. Cerebral aneurysm wall thickness analysis using intraoperative microscopy: Effect of size and gender on thin translucent regions. J Neurointerv Surg 2013;5:201-6.

12. Meng H, Tutino VM, Xiang J, Siddiqui A. High WSS or low WSS? Complex interactions of hemodynamics with intracranial aneurysm initiation, growth, and rupture: Toward a unifying hypothesis. AJNR Am J Neuroradiol 2014;35:1254-62.

13. Cebral JR, Mut F, Weir J, Putman C. Quantitative characterization of the hemodynamic environment in ruptured and unruptured brain aneurysms. AJNR Am J Neuroradiol 2011;32:145-51.
14. Xiang J, Natarajan SK, Tremmel M, Ma D, Mocco J, Hopkins LN, et al. Hemodynamic-morphologic discriminants for intracranial aneurysm rupture. Stroke 2011;42:144-52.

15. Miura $Y$, Ishida F, Umeda $Y$, Tanemura $H$, Suzuki $H$, Matsushima S, et al. Low wall shear stress is independently associated with the rupture status of middle cerebral artery aneurysms. Stroke 2013;44:51921.

16. Cebral JR, Sheridan M, Putman CM. Hemodynamics and bleb formation in intracranial aneurysms. AJNR Am J Neuroradiol 2010;31:304-10.

17. Laith M. Kadasi., Walter C. Dent., and Adel M. Malek,. Colocalization of thin-walled dome regions with low hemodynamic wall shear stress in unruptured cerebral aneurysms. J Neurosurg 119:172-179, 2013

18. Boussel L, Rayz V, McCulloch C, Martin A, Acevedo-Bolton G, Lawton M, et al: Aneurysm growth occurs at region of low wall shear stress: patient-specific correlation of hemodynamics and growth in a longitudinal study. Stroke 39:2997-3002, 2008

19. Frösen J, Piippo A, Paetau A, Kangasniemi M, Niemelä M, Hernesniemi J, et al: Remodeling of saccular cerebral artery aneurysm wall is associated with rupture: histological analysis of 24 unruptured and 42 ruptured cases. Stroke 35:2287-2293, 2004

20. Shojima M, Oshima M, Takagi K, Torii R, Hayakawa M, Katada K, et al: Magnitude and role of wall shear stress on cerebral aneurysm: computational fluid dynamic study of 20 middle cerebral artery aneurysms. Stroke 35:2500-2505, 2004 